\title{
Ist die Doppik wirklich das geeignete Rechnungswesen?
}

\section{Kritischer Dialog:Wie überlegen ist die Doppik der Kameralistik?}

\author{
Wer die Finanzen einer öffentlichen Verwaltung über die doppische Buchführung steuert, läuft Gefahr, \\ das Gemeinwohl aus den Augen zu verlieren. Die Doppik scheint der Kameralistik keinesfalls nur über- \\ legen, sondern sie kann auch zu Fehlsteuerungen führen. So jedenfalls die Einschätzung der Autoren \\ dieses Beitrags, die damit auch zu einem offensichtlich notwendigen Dialog beitragen wollen.
}

E in Jahrhunderte währender Kampf zwischen der kameralistischen und doppischen Buchführung scheint entschieden. Allein in dieser Zeitschrift konnte man seit Anfang 2000 jährlich mindestens ein Dutzend positiver bis euphorischer Beiträge und Erfolgsberichte zu den Errungenschaften der Doppik und leidenschaftliche Erklärungen namhafter Professoren finden, in denen der Systemwechsel als alternativlos beschrieben wird. Ein Blick in die Geschichte zeigt, dass der Versuch, öffentliche Institutionen über die Doppik zu steuern, eigentlich nicht neu ist: Bereits im 17. Jahrhundert sind Bemühungen in Frankreich und den Niederlanden, im 18. Jahrhundert in Österreich nachweisbar. Nach kurzen Ausflügen kehrte man aber zur Kameralistik zurück und setzte auf ihre Fortentwicklung.

\section{Produlte, Kosten-Leistungs- Rechnung und Bilanzen.}

Seit Mitte der 1990er Jahre ist nun - getrieben von schwierigen Haushaltslagen die Einführung der Doppik Teil der Verwaltungsmodernisierung (New Public Management). Budgetierung und Dezentralisierung, Einführung der Kosten-Leistungs-
Rechung (KLR) und eben die Einführung der Doppik sind zu den großen operativen Themen der deutschen Verwaltung geworden. Die Budgetierung wurde dankbar aufgenommen und erweist sich heute zusammen mit Deckungsfähigkeiten und Übertragbarkeiten als durchaus effektivitätssteigernd. Auch die Dezentralisierung, also das Zusammenführen von Entscheidung und Verantwortung, entspricht dem heutigen Führungsverständnis.

Schwierig wird es hingegen bei der KLR. Die Definition von Produkten, Kennzahlen und Leistungen für Sachverhalte, die oftmals gesetzlicher Auftrag und wenig bis kaum beeinflussbar sind, hat viele zum Verzweifeln und auch zum Aufgeben gebracht. Diejenigen, die eine KLR eingeführt hatten, haben sie meist auf das „K“ reduziert, da die Diskussionen über das „L“ kaum sinnvoll zu führen waren. Zudem braucht man ein Ersatzinstrument für die Budgetplanung, die zuvor über kamerale Haushaltsposten stattfand. Und so fristet die KLR ein Dasein als Produkt-Darstellung der Kosten, eine Inputsteuerung durch die Hintertür: von Entscheidern zu oft geschmäht und von den Mitarbeitern/innen meist für unsinnig befunden.

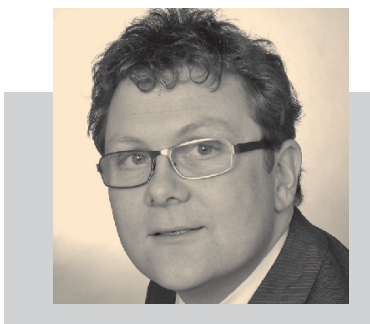

Dr. Timo Meynhardt ist Managing Director am Center for Leadership and Values in Society der Universität St. Gallen

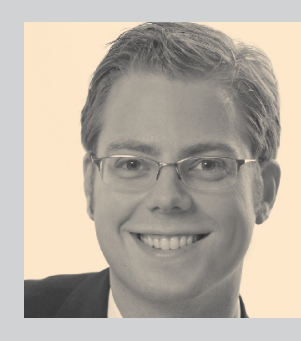

\section{Eric Schulze} ist Doktorand am Center for Leadership and Values in Society der Universität St. Gallen 
Anstatt aber die KLR grundsätzlich in Frage zu stellen, wird angemahnt, dass eine KLR ohne eine doppelte Buchführung nicht wirken kann und daher die Einführung der Doppik zwingend notwendig ist. Nur so seien der periodengerechte Ressourcenverbrauch und das transparente und eineindeutige Rechnungswesen garantiert. Mitten in dieser Phase befinden sich zurzeit die Kommunen und Bundesländer wie Hessen und Hamburg.

\section{Die Tugenden}

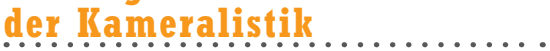

Um die alte Kameralistik ist es hingegen ruhig geworden. Wer will sich denn schon mit „Dezemberfieber“, „Punktlandung“ und „Haushaltstöpfchen“ identifizieren? Mit dieser Beschreibung wird man der Kameralistik aber nicht gerecht, denn manch eine Tugend schlummert in ihr:

- Das kameralistische System steht nicht im Gegensatz zu Budgetierung oder Dezentralisierung. Die Realisierung von Budgetierung, Deckungsfähigkeiten, Mittelübertragung und Zielvereinbarungen zeigt, wie man durchaus in einer Kameralistik neue Anreize zur Effektivitätssteigerung und Wirkungsorientierung setzen kann.

- Die Kameralistik ist ein Ein- und Auszahlungssystem: „Was zählt, ist das, was in die Kasse kommt.“ Die Privatwirtschaft nennt das „Cash-Flow“. Börsenanalysten ziehen diesen als wichtige Kenngröße heran, wenn zwar in Bilanzen Gewinne geschrieben werden, denen aber keine konkrete Einzahlung gegenübersteht (z. B. bei offenen Forderungen). Warum sollte also das, was in der Privatwirtschaft für viele Analysten gerade seit dieser Finanzkrise wichtiger ist als der Bilanzgewinn, für den Staat als antiquiert gelten?

- Die Kameralistik unterliegt - anders als die Doppik - nicht dem Prinzip des Gläubigerschutzes. Ein Staat kann fast nicht „pleite“ gehen. Anders als Privatunternehmen hat er fast grenzenlose Möglichkeiten, seine Einnahmen zu erhöhen (Steuererhöhung), Ausgaben zu senken (Leistungskürzung) oder Werte zu schaffen (Aktivierungen). Die sinnvollen Regelungen zur Deckung von Fremdkapital durch konservativ bewertete Aktivposten in der Privatwirtschaft sind für einen Staat weder handlungsleitend noch zielführend.

- Das kameralistische System ist für politische Entscheidungsträger/innen nachvollziehbar. Auch wenn viele kamerale Haushalte bereits Telefonbuchstärke erreicht haben, so ist das zugrunde liegende Prinzip von Einzahlung und Auszahlung für die Volksvertreter/innen verständlich. Die Frage lautet doch: „Haben wir die Mittel, um eine neue Turnhalle für 10 Mio. Euro zu bauen?“ und ist damit analog einer privaten Entscheidung: „Habe ich das Geld für einen neuen Fernseher?“. In der doppischen Welt lautet die Frage nun anders: „Wollen wir eine Turnhalle für 500.000 Euro über 20 Jahre?“Wie günstig, denkt sich da einer, und das Geld ... ja, das kommt aus der Stadtkasse, so lange das Jahresergebnis nur stimmt.

Wenn jetzt aber die Kameralistik doch nicht so schlecht sein soll, wie konnte es denn dann zur Anhäufung der Schulden und Verpflichtungen kommen? Die Antwort auf diese Frage liegt in der Tat beim periodengerechten Ressourcenverbrauch, der Paradedisziplin der Doppik.

\section{Die Mär von der}

d.oppischen Ựerlegenheit....

Was ist ein periodengerechter Ressourcenverbrauch? Einfach zu erklären ist das bei der Pension: Wenn ein Staatsdiener heute arbeitet, erwirbt er sich einen Pensionsanspruch, der aber erst bei Rentenantritt fällig wird. Periodengerecht müsste man also heute bereits das Geld beiseite legen oder - buchhalterisch gesprochen - die Forderung der Beamten/innen an den Staat in die Bücher übernehmen. Diese Logik ist sinnvoll, da es gegen unser Gerechtigkeitsempfinden verstößt, wenn heutige Generationen auf Kosten der nachfolgenden leben. Unbestreitbar ist auch, dass die Doppik diese Periodenabgrenzung abbilden kann, die Kameralistik aber nicht. Es sieht nach einem Sieg der Doppik auf voller Linie aus - aber schauen wir uns doch mal die großen
Blöcke der Generationen-Ungerechtigkeit näher an:

- Schulden: Der Schuldenberg der öffentlichen Haushalte ist in beiden Systemen gleichermaßen transparent. Weder Transparenz noch eingeführte Grenzmechanismen zur Staatsverschuldung (Neuverschuldung geringer Investitionen) haben das verhindern können. Weder war es der Wille der Politik noch der eindeutige Wille des Volkes, keine Schulden aufzunehmen. Zudem glaubte man lange an die Wirkung staatlicher Ausgabenpolitik. Es ist aber völlig klar, dass dem Grunde nach nicht mehr Geld ausgegeben werden darf als eingenommen wird - also eine einfache Ein- und Auszahlungsregelung und bewusst ohne Betrachtung von Investitionen, Abschreibungen etc. Der Schuldenberg als Periodenvergehen Nummer 1 ist in beiden Systemen abbildbar. Im kameralen System bleibt es aber ein „Berg ohne Gegenberg“, was die Lage $u$. U. noch etwas dramatischer darstellt.

- Pensionslasten: Die Pensionslasten bilden mit Abstand die größte Position der versteckten Periodenvergehen. Folgt man dem Vorsorgebericht der Bundesregierung von 2005, so steht bis 2050 mindestens eine Verdopplung der Pensionsausgaben an. Die Pensionsansprüche in Deutschland überschreiten die Billionengrenze. In einer doppischen Bilanz werden diese Pensionsansprüche transparent. Doch auch in der kameralen Bundesrepublik war man sich der Thematik durchaus bewusst. Die Zahlen waren in der Größenordnung auch bekannt, nur hat das nicht zu einem veränderten Verhalten geführt. Auch in einem kameralen System könnte man die Pensionslasten periodengerecht abbilden durch die Einrichtung von Pensionsfonds, die analog einer kapitalgedeckten Rentenversicherung eine direkte Kapitalspeisung durch den Arbeitgeber (Staat) erfordern. Es bleibt festzuhalten, dass ein kamerales System durchaus eine periodengerechte Abrechnung der Pensionen erlaubt. 


\section{- Investitionsstau bei der öffentlichen} Infrastruktur: Ein weiteres Periodenvergehen besteht darin, die im eigenen Verantwortungsbereich liegende Infrastruktur nicht angemessen zu pflegen, auf Kosten vergangener Investitionen zu leben und so ein strukturelles Haushaltsdefizit zu verschleiern. Diese Vergehen werden in der Doppik (in der Abschreibungen auf Investitionsgüter fällig werden) eindeutig sichtbar. Doch führt diese Sichtbarkeit in den Zahlen zu mehr politischem Entscheidungswillen als die stetige Auseinandersetzung mit den Bürgern/innen? Wird eine doppisch geführte Stadt trotz leerer Kassen die Infrastruktur instand setzen oderwie Privathaushalte und Unternehmen - das eine oder andere auf das nächste Jahr verschieben? Sie wird Investitionen von Fall zu Fall betrachten und damit aktiv Prioritäten setzen, anstatt der Buchhaltung den Job der Politik zu überlassen.

- Unterlassene Investitionen: Die Doppik ist ein an den Realwerten ausgerichtetes System: Unterlassene Investitionen, eine nicht gebaute Autobahn, eine nicht gebaute Turnhalle werden in beiden Systemen nicht erkannt. Vorteil der Kameralistik ist allerdings, dass sie die saldierte Darstellung staatlicher Investitionstätigkeit für sich gar nicht erst in Anspruch nimmt. Diese Versteifung auf materielle Realwerte, die auch der gerade abgelösten Neuverschuldungsregel zugrunde lag, begrenzt den Investitionsbegriff staatlichen Handelns künstlich. So kommt es, dass auch ein unnützer Transrapid einfach werterhaltender für die staatliche Bilanz wäre als der Ausbau der frühkindlichen Bildung, die ja leider einfach nur Geld kostet und Bilanzwerte vernichtet - welch ein Glück, dass staatliche Großbauprojekte in der Doppik einen strammen Befürworter haben. Die verpassten Chancen stehen dabei leider außerhalb der Bilanz.

- Verkauf des Tafelsilbers: Das letzte große Periodenvergehen ist der Verkauf staatlicher Beteiligungen zur Deckung eines strukturellen Defizits. Der Verkauf von Stadtwerken und Wohnungsbauge- sellschaften ist ein gutes Beispiel. Auch die Doppik kann solche politischen Entscheidungsprozesse nicht verhindern. Wohl aber stehen in der doppischen Bilanz den städtischen Wohnungen die Schulden auf der anderen Bilanzseite gegenüber. Mit kühler Transparenz ließe sich ausrechnen, dass die Wohnungen nicht die Rendite abwerfen, die auf der anderen Seite für die städtischen Schulden entrichtet werden, und dass daher ein Verkauf zwingend geboten ist. Das ist vergleichbar mit einem Unternehmen, das eine schwächelnde Sparte zum Verkauf anbietet. So wichtig diese Diskussion ist, so reduziert sie doch staatliches Eigentum auf eine Kapitalrendite und erschwert die ganzheitliche Diskussion über den Wert staatlicher Beteiligungen für das Gemeinwohl. Die Kameralistik lädt ein zum Verkauf, weil das Vergehen in den Büchern nicht auffällt; die Doppik lädt ein zum Verkauf, weil es die Zahlen gebieten. Beide Systeme können dem Staat nicht dabei helfen zu entscheiden, welches Tafelsilber er behalten soll. Das ist und bleibt ureigenste Aufgabe des politischen Prozesses.

\section{Besser doppelt nachdenken...}

Was könnte man den Zweiflern nun antworten? Zunächst, dass sie sich nicht von der Zahlenakrobatik ihrer Finanzexperten zu stark beeindrucken lassen sollen. Es ist ihre Pflicht, Erklärungen der Fachleute einzufordern, die auch für die Volksvertreter/innen verständlich sind. Öffentliche Haushaltsführung ist keine Raketenwissenschaft! Vor allem sollten sie darauf achten, dass das Rechnungswesen kein Selbstzweck wird und keinesfalls Organisationsstrukturen allein wegen eines neuen Rechnungswesens angepasst werden. Die Arbeit der Finanzverantwortlichen muss vielmehr als Dienstleistung betrachtet werden, der eine Unterstützungsfunktion bei der „Wertschöpfung zum Gemeinwohl“ zukommt. Dabei lassen sich wesentliche Wirkungsbereiche gerade nicht über finanzielle Größen fassen. Was betriebswirtschaftlich sinnvoll ist, muss noch lange nicht poli- tisch akzeptabel oder moralisch vertretbar sein. Gerade in der öffentlichen Verwaltung darf das ökonomische Kalkül nicht einseitig dominant werden. Genau das aber wird durch die Doppik systematisch gefördert.

Die Doppik kann genauso wenig wie die Kameralistik ein Volk und seine Regierenden daran hindern, „über ihre Verhältnisse“ zu leben. Wer denkt, durch die Einführung der Doppik seinen verantwortungsvollen Umgang mit den Ressourcen zu demonstrieren, irrt gewaltig. Mit einer Doppikeinführung kann man den Verwaltungsapparat lange und kräftezehrend beschäftigen, doch die Hausaufgaben und die eigentlichen Probleme werden dadurch nicht erledigt. Vielmehr führt die Engführung der doppelten Buchführung auf monetäre Größen zu erheblichen Fehlsteuerungsanreizen. Der vielschichtige Aktivposten „Gemeinwohl“ kann nur unzureichend durch einen Passivposten „Geld“ abgebildet werden. Keines der geschilderten Periodenvergehen hätte allein durch die Doppik verhindert werden können. Die Doppik ist bei den entscheidenden Fragen einer (erweiterten) Kameralistik nicht überlegen.

Das große Verdienst der Doppik liegt sicher in einer stärkeren Sensibilisierung für einen gerechten Ressourcenverbrauch und damit für zukünftige Belastungen. Der Systemwechsel von der Kameralistik zur Doppik hat jedoch keinen direkten Einfluss auf eine erhöhte Generationengerechtigkeit oder eine verbesserte „Wirtschaftlichkeit“ von politischen Entscheidungen. Weder Doppik noch Kameralistik sind denkende oder entscheidende Systeme. Sie entlasten die politischen Entscheidungsträger/innen nicht von ihren Entscheidungspflichten.

Die aktuelle Faszination für die Doppik ist also nicht gerechtfertigt. Die Protagonisten der Doppik sind bisher stichhaltige Argumente schuldig geblieben, warum im öffentlichen Sektor eine erweiterte Kameralistik nicht zielführender wäre. Kann man aus der Geschichte wirklich nur lernen, dass man aus ihr nichts lernen kann?

Weitere Informationen zum Thema erhalten Sie bei Timo Meynhardt, E-Mail: timo.meynhardt@unisg.ch. 